Commun. Korean Math. Soc. 20 (2005), No. 1, pp. 51-62

\title{
BOUND FOR 2-EXPONENTS OF PRIMITIVE EXTREMAL MINISTRONG DIGRAPHS
}

\author{
Sang Gu Lee and Jeong Mo Yang
}

\begin{abstract}
We consider 2-colored digraphs of the primitive ministrong digraphs having given exponents. In this paper we give bounds for 2-exponents of primitive extremal ministrong digraphs.
\end{abstract}

\section{Introduction}

We use the notation and terminology for digraphs as are in [4]. In this paper we let $D=(V, E)$ be a digraph and $\mathcal{D}$ be a 2-colored digraph of $D=(V, E)$. A nonnegative square matrix $A$ is primitive provided there is a nonnegative integer $k$ such that $A^{k}$ is entrywise positive (some authors say strictly positive), denoted by $A^{k} \gg 0$. If $A$ is primitive, the smallest integer $k$ such that $A^{k}$ has only positive entries is called the exponent of $A$, denoted by $\exp (A)$.

As defined in [6], a positive discrete homogeneous 2D-system is described by the equation.

(1.1) $x(h+1, k+1)=A x(h, k+1)+B x(h+1, k), h, k \in \mathbb{Z}, h+k \geq 0$,

where $A$ and $B$ are $n$ by $n$ nonnegative matrices and the initial conditions $x(h,-h)(h \in \mathbb{Z})$ are nonnegative $n$ by 1 vectors. System (1.1) is called the $2 \mathrm{D}$-system associated with the nonnegative matrix pair $(A, B)$. Positive discrete homogeneous 2D-dynamical systems are used in [5] to discretely model diffusion processes. A component of the vector $x(h, k)$ typically represents a quantity such as pressure, concentration or density at a particular site along a stream. We can view this stream as flowing left-to-right along the line $y=-x$. The points $(h,-h)(h \in \mathbb{Z})$ correspond to the discrete sites $h(h \in \mathbb{Z})$ along the stream. The vector

Received May 19, 2004.

2000 Mathematics Subject Classification: 05C20, 05C50.

Key words and phrases: Ministrong digraphs, 2-primitive, 2-exponent.

This work is supported by Faculty Research Fund at Sungkyunkwan University 2003 and $\mathrm{Com}^{2} \mathrm{MaC}-\mathrm{KOSEF}$. 
$x(h, k)$ represents the conditions at site $h$ after $h+k$ time-steps. Thus, $x(h,-h)$ describes the initial conditions at site $h$, the vector $x(h,-h+1)$ describes the conditions at site $h$ after 1 time-step, etc. Note that by setting $t=h+k+1$, the equation (1.1) indicates that conditions at site $h+1$ after $t+1$ time-steps are determined in a linear, time and location autonomous fashion from the conditions at site $h+1$ after $t$ time-steps and the conditions at site $h$ after $t$ time-steps. Thus, at each time-step the conditions of a site are determined by its previous conditions and the conditions of the site directly upstream from it [8].

Definition 1.1. [8] For nonnegative integers $h$ and $k$, the $(h, k)$ Hurwitz product, $(A, B)^{(h, k)}$, of $A$ and $B$ is the sum of all matrices that are a product of $h A$ 's and $k B$ 's.

For example, $(A, B)^{(1,0)}=A$ and

$$
(A, B)^{(2,2)}=A^{2} B^{2}+A B A B+A B^{2} A+B A^{2} B+B A B A+B^{2} A^{2} \text {. }
$$

Define the pair $(A, B)$ of nonnegative matrices to be 2-primitive provided there exist nonnegative integers $h$ and $k$ such that $h+k>0$ and $(A, B)^{(h, k)} \gg 0$. The 2-exponent of the primitive pair $(A, B)$ of matrices is defined to be the minimum value of $h+k$ taken over all pairs $(h, k)$ such that $(A, B)^{(h, k)} \gg 0$. We write $\exp (A, B)$ for the 2-exponent of the pair $(A, B)$.

In [6], it is shown that the nonnegative matrix pair $(A, B)$ has both $A$ and $B$ nonzero and is primitive if and only if the solutions to (1.1) are eventually strictly positive. This shows that the definition of 2primitivity of matrix pairs truly generalizes the primitivity for a single nonnegative matrices.

A two-colored digraph is a digraph whose arcs are colored red or blue. We allow loops and multiple colored arcs from $i$ to $j$. There is a natural correspondence between two-colored digraphs and nonnegative matrix pairs. With each two-colored digraph $\mathcal{D}$ we associate a pair $(A, B)$ of $(0,1)$-matrices where the $(i, j)$-entry of $A$ is 1 if and only if there is a red arc from $i$ to $j$, and the $(i, j)$-entry of $B$ is 1 if and only if there is a blue arc from $i$ to $j$. For each pair $(A, B)$ of nonnegative $n$ by $n$ matrices, we associate the 2-colored digraph, $\mathcal{D}(A, B)$, with vertices $1,2, \ldots, n$, a red arc from $i$ to $j$ if $a_{i j}>0$ and a blue arc from $i$ to $j$ if $b_{i j}>0$. An $(h, k)$-walk from $i$ to $j$ in $\mathcal{D}$ is a walk from $i$ to $j$ consisting of $h$ red arcs, and $k$ blue arcs. One can easily show that the $(i, j)$-entry of $(A, B)^{(h, k)}$ is strictly positive if and only if there is an $(h, k)$-walk in $\mathcal{D}(A, B)$ from $i$ to $j$. Given a walk $w$ in $\mathcal{D}$, we write $r(w)$ and $b(w)$ for the numbers of 
red and blue arcs that $w$ has in it, and we call the column vector

$$
\left[\begin{array}{l}
r(w) \\
b(w)
\end{array}\right]=(r(w), b(w))
$$

the composition of $w$. The two-colored digraph $\mathcal{D}$ is strongly connected provided for each pair $(i, j)$ of vertices there is a walk in $\mathcal{D}$ from $i$ to $j$. The matrix pair $(A, B)$ is 2-primitive if and only if there exist nonnegative integers $h$ and $k$ with $h+k>0$ such that for each pair $(i, j)$ of vertices there exists an $(h, k)$-walk in $\mathcal{D}$ from $i$ to $j$. We say the 2 -colored digraph $\mathcal{D}$ is 2-primitive provided the associated matrix pair $(A, B)$ is 2-primitive and the 2-exponent of $\mathcal{D}$ is defined to be the 2-exponent of $(A, B)$. The definition for cycle matrix of $\mathcal{D}$ is given as following.

Definition 1.2. [8] Let $\mathcal{D}$ be a 2-colored digraph and let $C=$ $\left\{\gamma_{1}, \gamma_{2}, \ldots, \gamma_{c}\right\}$ be the set of all cycles of $\mathcal{D}$. Set $M$ to be the 2 by $c$ matrix whose $i$ th column is the composition of $\gamma_{i}$, and $\langle M\rangle$ to be the additive subgroup of $\mathbb{Z}^{2}$ generated by the columns of $M$. We call $M$ the cycle matrix of $\mathcal{D}$.

In Definition 1.2, $\langle M\rangle=\mathbb{Z}^{2}$ implies that $M$ has at least two linearly independent columns. Moreover, since each closed walk of $\mathcal{D}$ can be decomposed into cycles of $\mathcal{D}$, the composition of each closed walk of $\mathcal{D}$ belongs to $\langle M\rangle$.

Let $n \leq c$. The content of the $n$ by $c$ matrix $M$, denoted by content $(M)$, is defined to be 0 if the rank of $M$ is less than $n$ and to be the greatest common divisor of the determinants of the $n$ by $n$ submatrices of $M$, otherwise.

Definition 1.3. [4] A strongly connected digraph $D$ is called ministrong (or minimally strongly connected) if each digraph obtained from $D$ by the removal of an arcs is not strongly connected.

By definition, a ministrong digraph has no loops. Each arc of a digraph $D$ corresponds to 1 in its adjacency matrix $A$. Thus the removal of an arc in $D$ means the replacement of a 1 with a 0 in $A$. An irreducible matrix $A$ is called nearly reducible provided each matrix obtained from $A$ by replacing of a 1 with a 0 is a reducible matrix. Thus the digraph $D$ is ministrong if and only if its adjacency matrix $A$ is nearly reducible. More generally we say that an arbitrary matrix $A$ of order $n$ is nearly reducible if its digraph $D$ is ministrong [4].

In section 2, we introduce some known results on ministrong digraphs and the 2-primitivity of 2-colored digraphs. 


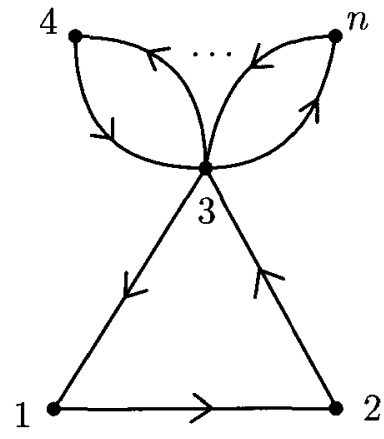

(a) $\exp (D)=6$

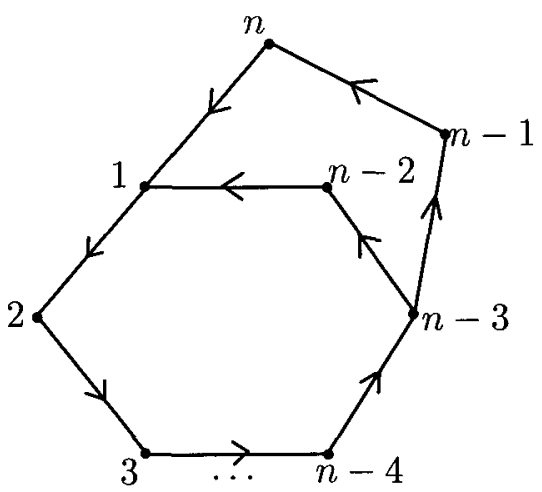

(b) $\exp (D)=n^{2}-4 n+6$

FIgURE 1. Primitive ministrong digraphs with $6 \leq$ $\exp (D) \leq n^{2}-4 n+6$

In section 3 , we consider a primitive ministrong digraph $D$ with the largest exponent $n^{2}-4 n+6$ and find bounds for 2-exponent of $\mathcal{D}$ and $(h, k)$-walks from $i$ to $j$ for all $i, j \in V(\mathcal{D})$ satisfying our bounds for 2-exponent. Also, we find the 2-exponent of 2-primitive ministrong digraph on the primitive ministrong digraph with the least exponent 6 .

Many applications of the 2-primitivity can be found in $[2,5,8]$.

\section{Preliminaries}

Let $D=(V, E)$ be a primitive ministrong digraph of order $n$. In [3], Brualdi and Ross showed the following lemma.

LEMMA 2.1. [3] Let $D$ be a primitive ministrong digraph with vertices. Then

$$
6 \leq \exp (D) \leq n^{2}-4 n+6
$$

with equality if and only if $D$ is isomorphic to the digraph in Figure 1.

In [7], Ross showed that $\exp (D) \leq n+s(n-3)$ for any ministrong digraph $D$ with girth $s$ and gave a digraph with equality.

The following Propositions 2.2 and Remark 1 are important for testing whether the nonnegative matrix pair $(A, B)$ is primitive or not.

Proposition 2.2. [8] Let $(A, B)$ be a pair of $n$ by $n$ nonnegative matrices with both $A$ and $B$ nonzero and let $M$ be the cycle matrix of $\mathcal{D}(A, B)$. Then the following are equivalent: 
(a) $(A, B)$ is primitive,

(b) $\mathcal{D}(A, B)$ is strongly connected and $\langle M\rangle=\mathbb{Z}^{2}$,

(c) $\mathcal{D}(A, B)$ is strongly connected and content $(M)=1$.

One may rephrase this proposition as follows:

REMARK 1. A matrix pair $(A, B)$ is primitive if and only if the greatest common divisor of 2 by 2 minors of the cycle matrix $M$ of $\mathcal{D}(A, B)$ is 1 .

Recently, Beasley and Kirkland showed the following proposition.

Proposition 2.3. [1] If $D$ is a primitive directed graph, then there is a 2-coloring of $D$ with which the corresponding 2-colored digraph $\mathcal{D}$ is 2-primitive.

Shader and Suwilo gave the bounds on the 2-exponent of each 2primitive Wielandt digraphs $\mathcal{D}$ as $2 n^{2}-4 n+1 \leq \exp (\mathcal{D}) \leq 2 n^{2}-3 n+1$ and then gave examples of 2-primitive digraphs with 2-exponent $\left(n^{3}-\right.$ $\left.2 n^{2}+1\right) / 2$ for each odd integer $n \geq 5$ and $\left(n^{3}-5 n^{2}+7 n-2\right) / 2$ for each even integer $n \geq 6$ in [8].

\section{Exponents of primitive extremal ministrong digraphs}

In this section we consider a primitive ministrong digraph $D$ with the largest exponent on $n$ vertices as one can see $(b)$ of Figure 1. After we consider a 2-coloring of arcs on $D$, we show that the 2-colored $\mathcal{D}$ is 2 -primitive and that $2 n^{2}-8 n+7 \leq \exp (\mathcal{D}) \leq 2 n^{2}-5 n+3$. Also, we give a 2-coloring to arcs on a primitive ministrong digraph with the smallest exponent 6 on $n$ vertices as one can see $(a)$ of Figure 1. Then it has 2-exponent 12 .

We can find an upper bound for a 2-exponent of 2-primitive digraphs from Lemma 3.1.

LEMma 3.1. [8, Lemma 1] Let $\mathcal{D}$ be a strongly connected, 2-colored digraph with cycle matrix $M$. For each pair $(i, j)$ of vertices let $p_{i j}$ be a path from $i$ to $j$ and let $w$ be a closed walk that goes through each vertex of $\mathcal{D}$. Suppose that $\mathbf{z}=\left(z_{1}, z_{2}, \ldots, z_{c}\right)^{T}$ is a nonnegative, integer vector such that each system

$$
M \mathbf{x}_{i j}=\left[\begin{array}{l}
r\left(p_{i j}\right) \\
b\left(p_{i j}\right)
\end{array}\right]
$$


has an integer solution $\mathbf{x}_{i j}$ with $\mathbf{z} \geq \mathbf{x}_{i j}$. Then $\mathcal{D}$ is 2-primitive and $\exp (\mathcal{D}) \leq h+k$, where $h$ and $k$ are defined by

$$
\left[\begin{array}{l}
h \\
k
\end{array}\right]=\left[\begin{array}{l}
r(w) \\
b(w)
\end{array}\right]+M \mathbf{z}
$$

We start with two-colored digraphs obtained by coloring arcs of the primitive ministrong digraph $D$ which has the largest exponent $n^{2}-4 n+$ 6 in Lemma 2.1. We give a bound for 2-exponents of the two-colored digraphs of $D$.

THEOREM 3.2. Let $D$ be a primitive ministrong digraph on $n$ vertices with the largest exponent $n^{2}-4 n+6$. Let $\mathcal{D}$ be a 2-primitive ministrong digraph on $n$ vertices with at least one red and one blue arc. Then

$$
2 n^{2}-8 n+7 \leq \exp (\mathcal{D}) \leq 2 n^{2}-5 n+3
$$

First equality is obtained by the coloring $D$ like the Figure 2-(b) (blue arc is only in $(n-2)$-cycle). The second equality is obtained by the coloring $D$ like the Figure 2-(a) (one blue arc is in $(n-1)$-cycle and the other is in $(n-2)$-cycle).

Proof. We give red and blue colors to the $\operatorname{arcs}$ of $D$. Let $\mathcal{D}$ be the two-colored ministrong digraph of $D$ and assume that $\mathcal{D}$ has at least one red and one blue arc.

Let $M=\left[\begin{array}{cc}n-1-a & n-2-b \\ a & b\end{array}\right]$ be a cycle matrix of $\mathcal{D}$. Since $\operatorname{det} M \equiv 2 a-b \equiv 1(\bmod n)$ for $\mathcal{D}$ to be 2 -primitive, we have $a=b=1$. One can take $a=n-2$ and $b=n-3$, but this is just changing color between red and blue. So this argument has no loss of generality because even though we choose any other $a \neq 1, b \neq 1$ which satisfy $2 a-b \equiv$ $1(\bmod n)$, it is easy to check that there is no difference in getting the upper bound for 2-exponent. So one can take the cycle matrix $M$ of $\mathcal{D}$

$$
M=\left[\begin{array}{cc}
n-2 & n-3 \\
1 & 1
\end{array}\right]
$$

and $\mathcal{D}$ is 2-primitive by Proposition 2.2 .

This cycle matrix $M$ implies that $(n-1)$-cycle of $\mathcal{D}$ contains exactly one blue arc. There are two possibilities. We say $\mathcal{D}$ is of type I (Figure 2-(a)), if the blue arc is one of $(n-3) \rightarrow(n-1)$ and $(n-1) \rightarrow n$ and $n \rightarrow 1$, and $\mathcal{D}$ is type II (Figure 2-(b)), otherwise. If $\mathcal{D}$ is type I, one of $(n-3) \rightarrow(n-2)$ and $(n-2) \rightarrow 1$ is blue. If $\mathcal{D}$ is type II, the arcs $(n-3) \rightarrow(n-2)$ and $(n-2) \rightarrow 1$ are both red. 

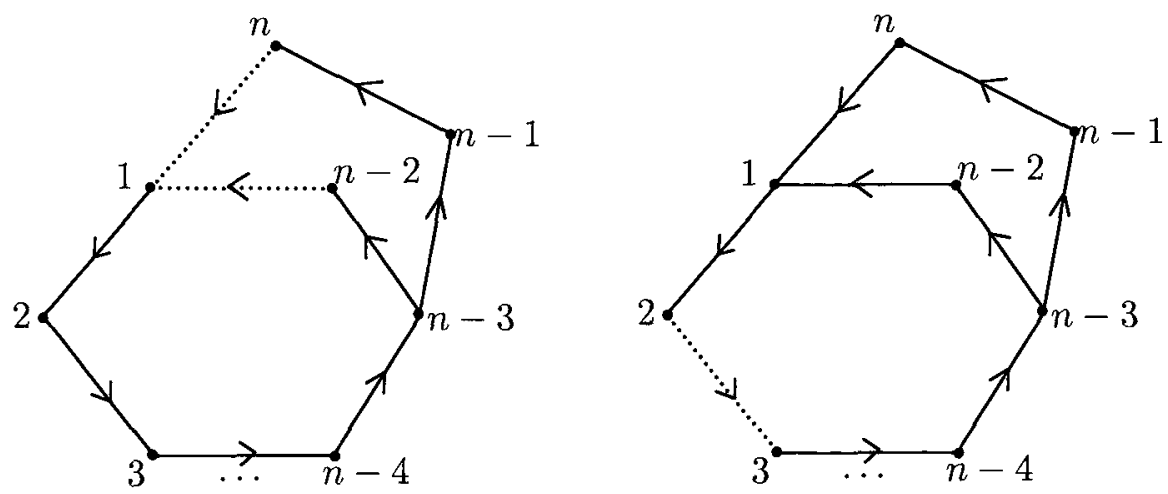

Thick arcs: red / Dotted arcs: blue

(a) Type I-upper bound case

(b) Type II-lower bound case

Figure 2. The 2-primitive ministrong digraphs

Let $p_{i j}$ be the path from $i$ to $j$ in $\mathcal{D}$. Let

$$
\left[\begin{array}{l}
u \\
v
\end{array}\right]=M^{-1}\left[\begin{array}{l}
r\left(p_{i j}\right) \\
b\left(p_{i j}\right)
\end{array}\right]
$$

for $\left[\begin{array}{l}r\left(p_{i j}\right) \\ b\left(p_{i j}\right)\end{array}\right]$ as is in (1.2). Note that for each pair of vertices $i$ and $j$ of $\mathcal{D}$ there exists a path $p_{i j}$ from $i$ to $j$, with $r\left(p_{i j}\right) \leq n-2$ and $b\left(p_{i j}\right) \leq 1$. After analyzing all paths $p_{i j}$, we know that $\mathbf{x}_{i j}:=(u, v)^{T} \leq$ $(n-2, n-2)^{T}$. Let $w$ be a closed walk that goes through each vertex in $V(\mathcal{D})$. In other words, $w$ is a combined walk from 1 to 1 using $(n-1)$ cycle and $(n-2)$-cycle. By Lemma 3.1,

$$
\begin{aligned}
{\left[\begin{array}{l}
h \\
k
\end{array}\right] } & =\left[\begin{array}{l}
r(w) \\
b(w)
\end{array}\right]+M \mathbf{z}\left(\text { with } \mathbf{z} \geq \mathbf{x}_{i j}\right) \\
& =\left[\begin{array}{c}
2 n-5 \\
2
\end{array}\right]+\left[\begin{array}{cc}
n-2 & n-3 \\
1 & 1
\end{array}\right]\left[\begin{array}{l}
n-2 \\
n-2
\end{array}\right] \\
& =\left[\begin{array}{c}
2 n^{2}-7 n+5 \\
2 n-2
\end{array}\right] .
\end{aligned}
$$

Therefore, $\exp (\mathcal{D}) \leq h+k=2 n^{2}-5 n+3$. We will later find a $\left(2 n^{2}-\right.$ $7 n+5,2 n-2)$-walk between each pair $(i, j)$ of vertices of $\mathcal{D}$ in Remark 2.

Now we think the case of lower bound on the 2-exponent of $\mathcal{D}$ (Figure 2-(b)). Suppose that $(h, k)$ is a pair of nonnegative integers such that 
for all pairs $(i, j)$ of vertices there is an $(h, k)$-walk from $i$ to $j$. When $i=j$, we see that there exist nonnegative integers $u$ and $v$ with

$$
\left[\begin{array}{l}
h \\
k
\end{array}\right]=M\left[\begin{array}{l}
u \\
v
\end{array}\right] .
$$

This walk is the type of walk that are using only cycles. Next choose a blue arc on the $(n-1)$-cycle as like taking $i$ and $j$ to be the initial vertex and terminal vertex of the blue arc, respectively. Then the arcs of each walk from $i$ to $j$ can be decomposed into the arc from $i$ to $j$ and cycles. Hence,

$$
\left[\begin{array}{c}
h \\
k-1
\end{array}\right]=M \mathbf{z}
$$

has a nonnegative integer solution.

$$
\begin{aligned}
\mathbf{z} & =M^{-1}\left[\begin{array}{c}
h \\
k-1
\end{array}\right] \\
& =\left[\begin{array}{l}
u \\
v
\end{array}\right]+\left[\begin{array}{c}
n-3 \\
-n+2
\end{array}\right] \\
& =\left[\begin{array}{l}
u+n-3 \\
v-n+2
\end{array}\right] \geq 0
\end{aligned}
$$

Therefore,

$$
v \geq n-2 .
$$

Now choose a blue arc on the $(n-1)$-cycle as like taking $i$ and $j$ to be the terminal vertex and initial vertex of the blue arc, respectively. Since the only paths from $i$ to $j$ have composition either $(n-2,0)$ or $(n-3,0)$, either
(i) $\left[\begin{array}{c}h-(n-2) \\ k\end{array}\right]$
]$=M \mathbf{z} \quad$ or
(ii) $\left[\begin{array}{c}h-(n-3) \\ k\end{array}\right]=M \mathbf{z}$

In the case of (i), we have

$$
\begin{aligned}
0 \leq \mathbf{z} & =M^{-1}\left[\begin{array}{c}
h-(n-2) \\
k
\end{array}\right] \\
& =\left[\begin{array}{l}
u \\
v
\end{array}\right]-M^{-1}\left[\begin{array}{c}
n-2 \\
0
\end{array}\right] \\
& =\left[\begin{array}{l}
u-n+2 \\
v+n-2
\end{array}\right] .
\end{aligned}
$$

Therefore, $u \geq n-2$. Similarly, we have $u \geq n-3$ for the case (ii). Thus

$$
u \geq n-3,
$$


and

$h+k=\left[\begin{array}{ll}1 & 1\end{array}\right] M\left[\begin{array}{l}u \\ v\end{array}\right] \geq\left[\begin{array}{ll}n-1 & n-2\end{array}\right]\left[\begin{array}{l}n-3 \\ n-2\end{array}\right]=2 n^{2}-8 n+7$.

Therefore, we now have the interval for the 2-exponent of the 2-primitive ministrong digraph with the largest exponent;

$$
\exp (\mathcal{D}) \in\left[2 n^{2}-8 n+7,2 n^{2}-5 n+3\right]
$$

REMARK 2. We can find a $\left(2 n^{2}-7 n+5,2 n-2\right)$-walk of length $2 n^{2}-5 n+3$ from $i$ to $j$ for all $i, j \in V$, which is the upper bound for 2-exponent in Theorem 3.2, as follows:

If $(i, j)=(n, n)$, then the walk that starts at vertex $i$, goes to vertex 1 , goes $(n-1)$-times around the $(n-2)$-cycle, goes back to vertex $j$ and then goes $(n-2)$-times around the $(n-1)$-cycle has composition

$$
(n-1)\left[\begin{array}{c}
n-2 \\
1
\end{array}\right]+(n-1)\left[\begin{array}{c}
n-3 \\
1
\end{array}\right]=\left[\begin{array}{c}
2 n^{2}-7 n+5 \\
2 n-2
\end{array}\right] \text {. }
$$

Similarly, we can find a $\left(2 n^{2}-7 n+5,2 n-2\right)$-walk from $i$ to $j$ when $i=j$.

For the case of $(i, j)=(n-1, n)$, the walk that starts at vertex $i$, goes to 1 , goes $n$-times around the $(n-2)$-cycle, goes to vertex $j$ and goes $(n-2)$-times around the $(n-1)$-cycle has composition

$$
M\left[\begin{array}{c}
n-2 \\
n
\end{array}\right]+\left[\begin{array}{l}
1 \\
0
\end{array}\right]=\left[\begin{array}{c}
2 n^{2}-7 n+5 \\
2 n-2
\end{array}\right] .
$$

For the case of $(i, j)=(n, n-1)$, the walk that starts at vertex $i$, goes to 1 , goes $(n-2)$-times around $(n-2)$-cycle, goes to vertex $j$ and goes $(n-1)$-times around $(n-1)$-cycle has composition

$$
M\left[\begin{array}{l}
n-1 \\
n-2
\end{array}\right]+\left[\begin{array}{c}
n-3 \\
1
\end{array}\right]=\left[\begin{array}{c}
2 n^{2}-7 n+5 \\
2 n-2
\end{array}\right] .
$$

Otherwise, $p_{i j}$ contains vertices of the $(n-1)$-cycle and the $(n-2)$ cycle, and hence there is a walk that starts at vertex $i$, follows $p_{i j}$ to vertex $j$ and along the way and goes around the $(n-1)$-cycle $(n-1-u)$ times and the $(n-1)$-cycle $(n-1-v)$-times. Such a walk has composition

$$
\left[\begin{array}{l}
r\left(p_{i j}\right) \\
b\left(p_{i j}\right)
\end{array}\right]+M\left[\begin{array}{l}
n-1-u \\
n-1-v
\end{array}\right]=M\left[\begin{array}{l}
n-1 \\
n-1
\end{array}\right]=\left[\begin{array}{c}
2 n^{2}-7 n+5 \\
2 n-2
\end{array}\right]
$$

For all choices of $i, j \in V$, we can find a $\left(2 n^{2}-7 n+5,2 n-2\right)$-walk from $i$ to $j$. 
Theorem 3.3. Let $D$ be a primitive ministrong digraph on $n \geq 4$ with the smallest exponent 6 as in Lemma 2.1.(See (a) of Figure 1.) Let $\mathcal{D}$ be a 2-primitive ministrong digraph on $n \geq 4$ vertices. Then $\exp (\mathcal{D})$ is 12 .

Proof. As one can see (a) of Figure 1, $D$ has one 3-cycle and $(n-3)$ 2-cycles. So we now consider the submatrix of the cycle matrix of $\mathcal{D}$ as following.

$$
M=\left[\begin{array}{cc}
3-a & 2-b \\
a & b
\end{array}\right] .
$$

Since $\mathcal{D}$ is 2-primitive, $\operatorname{det}(M) \equiv 1$ which implies $a=b=1$. So we can give a coloring to arcs on $D$ as blue color to the arcs from 1 to 2 and from 3 to $k$ for $4 \leq k \leq n$ and red color to others. Then the 2-colored $\mathcal{D}$ has one $(2,1)$-cycle and $(n-3)(1,1)$-cycles. Let $M$ be the cycle matrix of $\mathcal{D}$,

$$
M=\left[\begin{array}{ll}
2 & 1 \\
1 & 1
\end{array}\right] .
$$

One can easily check all path $p_{i j}$ from $i$ to $j$ in $\mathcal{D}$ have lengths at most 3. A composition (reds, blues) of path $p_{i j}$ is one of $(1,0),(1,1),(1,2)$, $(2,0)$ and $(2,1)$. All $(h, k)$-walks from $i$ to $j$ in $\mathcal{D}$ are composed of the path $p_{i j}$ and cycles. Suppose that $(h, k)$ is a pair of nonnegative integers such that for all pairs $(i, j)$ of vertices there is an $(h, k)$-walk from $i$ to $j$. When $i=j$, we see that there exist nonnegative integers $u$ and $v$ with

$$
\left[\begin{array}{l}
h \\
k
\end{array}\right]=M\left[\begin{array}{l}
u \\
v
\end{array}\right]
$$

This walk is the type of walk that are using only cycles. Next take paths $p_{i j}$ from $i$ to $j$ for $i \neq j$. Then the arcs of each walk from $i$ to $j$ can be decomposed into $p_{i j}$ and cycles.

For a path $(2,0)$,

$$
\left[\begin{array}{c}
h-2 \\
k
\end{array}\right]=M \mathbf{z}
$$

has a nonnegative integer solution.

$$
\begin{aligned}
\mathbf{z} & =M^{-1}\left[\begin{array}{c}
h-2 \\
k
\end{array}\right] \\
& =\left[\begin{array}{l}
u \\
v
\end{array}\right]+\left[\begin{array}{c}
2 \\
-2
\end{array}\right] \\
& =\left[\begin{array}{l}
u-2 \\
v+2
\end{array}\right] \geq 0
\end{aligned}
$$


Therefore,

$$
u \geq 2
$$

Similarly, using all paths, we can easily check $(u, v)^{T} \geq(2,3)$. Therefore,

$$
\left[\begin{array}{l}
h \\
k
\end{array}\right]=M\left[\begin{array}{l}
u \\
v
\end{array}\right] \geq\left[\begin{array}{l}
7 \\
5
\end{array}\right]
$$

We now have $\exp (\mathcal{D})=12$ and one can find $(7,5)$-walks from $i$ to $j$ for all $i, j \in V(\mathcal{D})$.

Acknowledgements. The authors would like to thank the anonymous referee for a number of helpful suggestions.

\title{
References
}

[1] L. B. Beasley and S. Kirkland, $A$ note on $k$-primitive directed graphs, Linear Algebra Appl. 373 (2003), 67-74.

[2] A. Berman and R. Plemmons, Nonnegative Matrices in the Mathematical Sciences, Classics in Applied Mathematics, SIAM. Philadelphia, PA. 9 (1994).

[3] R. A. Brualdi and J. A. Ross, On the exponent of a primitive nearly reducible matrix, Math. Oper. Res. 5 (1980), no. 2, 229-241.

[4] R. A. Brualdi and H. J. Ryser, Combinatorial Matrix Theory. Encyclopedia of Mathematics and its Applications, Cambridge University Press, Cambridge, MA, 1991.

[5] E. Fornasini, A 2D systems approach to river pollution modelling, Multidimens. Systems Signal Process. 2 (1991), 233-255.

[6] E. Fornasini and M. Valcher, On the spectral and combinatorial structure of $2 D$ positive systems, Linear Algebra Appl. 245 (1996), 223-258.

[7] J. A. Ross, On the exponent of a primitive nearly reducible matrix II, SIAM J. Algebraic Discrete Methods 3 (1982), no. 3, 395-410.

[8] B. L. Shader and S. Suwilo, Exponent of nonnegative matrix pairs, Linear Algebra Appl. 363 (2003), 275-293.

\author{
Sang-Gu Lee \\ Department of Mathematics \\ Sungkyunkwan University \\ Suwon 440-746, Republic of Korea \\ E-mail: sglee@skku.cdu
}


Jeong-Mo Yang

Combinatorial and Computational Mathematics Center Pohang University of Science and Technology

Pohang 790-784, Republic of Korea

E-mail: jmyang@skku.edu 